\title{
MESOFAUNA DO SOLO EM REMANESCENTE DE CAATINGA, SANTANA DO IPANEMA, ALAGOAS
}

Jardel Estevam Barbosa dos Santos ${ }^{1 *}$; Anderson Marques Araújo do Nascimento1; Geovânia Ricardo dos Santos $^{1} ;$ Ana Beatriz da Silva ${ }^{1}$; Kallianna Dantas Araujo ${ }^{1}$

\author{
${ }^{1}$ Instituto de Geografia, Desenvolvimento e Meio Ambiente, Universidade Federal de Alagoas, Av. Lourival Melo Mota, s/n, Tabuleiro dos \\ Martins, Maceió, AL.
}

*Autor para correspondência: Jardel Estevam Barbosa dos Santos, jardelestevam@gmail.com

\begin{abstract}
RESUMO: Os organismos da mesofauna edáfica são importantes na decomposição da matéria orgânica, ciclagem de nutrientes e estruturação do solo, considerados bioindicadores de qualidade ambiental. Objetivou-se avaliar a abundância, riqueza, diversidade, equabilidade e constância da mesofauna invertebrada do solo, em área de remanescente de Caatinga, Santana do Ipanema, Alagoas. Foram realizadas coletas bimestrais, durante os meses de agosto de 2016 a fevereiro de 2017, em 20 parcelas para avaliação da mesofauna do solo. Foram utilizados anéis metálicos com diâmetro de $4,8 \mathrm{~cm}$ e altura de $5 \mathrm{~cm}$ para coleta de solo + serapilheira. Os anéis com as amostras de solo foram instaladas na bateria de extratores Berlese-Tullgren modificada para a extração dos organismos do solo por um período de 96 horas. Os grupos taxonômicos mais dominantes na área de Caatinga, confirmado pelos baixos índices de Shannon e Pielou são Collembola e Acarina, classificados como constantes em relação aos demais grupos taxonômicos que são inseridos na categoria acessória; O remanescente de Caatinga estudado encontra-se preservado fornecendo condições favoráveis à dinâmica dos organismos da mesofauna edáfica.
\end{abstract}

PALAVRAS-CHAVE: Semiárido, Organismos edáficos, Índices ecológicos.

\section{MESOFAUNA OF SOIL IN REMANESCENT OF CAATINGA, SANTANA DO IPANEMA, ALAGOAS}

\begin{abstract}
The organisms of the edaphic mesofauna are important in the decomposition of organic matter, nutrient cycling and soil structuring, considered bioindicators of environmental quality. The objective of this study was to evaluate the abundance, richness, diversity, and constancy of the invertebrate mesofauna of the soil, in the Caatinga remnant area, Santana do Ipanema, Alagoas. Bimonthly collections were carried out, during the months of August 2016 to February 2017, in 20 plots for evaluation of soil mesofauna. Metal rings with a diameter of $4.8 \mathrm{~cm}$ and a height of $5 \mathrm{~cm}$ were used to collect soil+litter. The rings with the soil samples were installed in the modified Berlese-Tullgren extractor battery for the extraction of soil organisms for a period of 96 hours. The most dominant taxonomic groups in the Caatinga area, confirmed by the low rates of Shannon and Pielou are Collembola and Acarina, classified as constant in relation to the other taxonomic groups that are inserted in the accessory category; The remainder of Caatinga studied is preserved providing conditions favorable to the dynamics of the organisms of the edaphic mesofauna.
\end{abstract}

KEYWORDS: Semiarid, Edaphic organisms, Ecological indexes.

\section{INTRODUÇÃO}

A mesofauna edáfica reúne os organismos invertebrados que vivem no solo ou passam parte do seu ciclo de vida nele e possuem tamanho corporal entre 0,2 - 2,0 mm de comprimento, incluindo os grupos Acarina (Ácaro), Collembola (Colêmbolo), Diplura (Dipluro),
Protura (Proturo), dentre outros (Almeida et al., 2013). Estes organismos desempenham funções importantes no solo, como a decomposição da matéria orgânica e a ciclagem de nutrientes, além de estruturação do solo, sendo considerados bioindicadores de qualidade ambiental (Berude et al., 2015). 
A composição da fauna edáfica está associada a diversos fatores biológicos, que interagem entre si e são sensíveis às condições edafoclimáticas (Korasaki et al., 2013). Esse conjunto de fatores afetam diretamente os organismos do solo, resultando nas variadas populações, dependendo do tipo de solo, vegetação e das condições microclimáticas (Berude et al., 2015).

Nas regiões Semiáridas é importante a realização de pesquisas que abordem a dinâmica da mesofauna edáfica e sua importância no funcionamento do ecossistema. Nesse sentido, objetivou-se avaliar a abundância, riqueza, diversidade, equabilidade e constância da mesofauna do solo, em área de remanescente de Caatinga, Santana do Ipanema, Alagoas.

\section{MATERIAL E MÉTODOS}

\section{Caracterização da área}

A pesquisa foi executada na Estação Experimental da EMATER (Empresa de Assistência Técnica e Extensão Rural), em Santana do Ipanema, Alagoas, Microrregião Geográfica de Santana do Ipanema e Mesorregião Geográfica do Sertão
Alagoano. A Sede do município encontra-se a 09²2'42" S e 37014'43" W (Alagoas, 2015).

O município está sob o domínio climático BSh-Tropical Semiárido, segundo a classificação de Köppen. Os solos predominantes no município são Neossolos Litólicos e Neossolos Regolíticos. O tipo de vegetação predominante é a Caatinga Hipoxerófila, com presença de áreas menores de Caatinga Hiperxerófila (Embrapa, 2012).

\section{Mesofauna edáfica}

A determinação da mesofauna do solo foi realizada em 20 parcelas amostrais, de $10 \times 10 \mathrm{~m}$, mediante coleta de amostras de solo+serapilheira utilizando anéis metálicos (diâmetro $=4,8 \mathrm{~cm}$ e altura $=5,0 \mathrm{~cm}$ ), a $5 \mathrm{~cm}$ de profundidade, na qual foram envolvidos em tecidos de filó e TNT, presos com liga de borracha (Figuras $1 \mathrm{~A}$ a $1 \mathrm{~F}$ ) e armazenados em caixa de isopor para serem transportados ao Laboratório de Ecogeografia e Sustentabilidade Ambiental- LabESA/ IGDEMA/UFAL, para serem instalados na bateria de extratores Berlese-Tullgren modificada para a extração dos organismos, durante 96 horas (4 dias).

Figura 1. Umedecimento do solo $(A)$ introdução do anel metálico no solo $(B)$, remoção da amostra $(C)$, armazenamento da amostra em tecido filó e TNT presos com liga de borracha (D), acondicionamento em caixa de isopor $(E)$, bateria de extratores Berlese-Tullgren modificado para extração da mesofauna edáfica $(F)$.
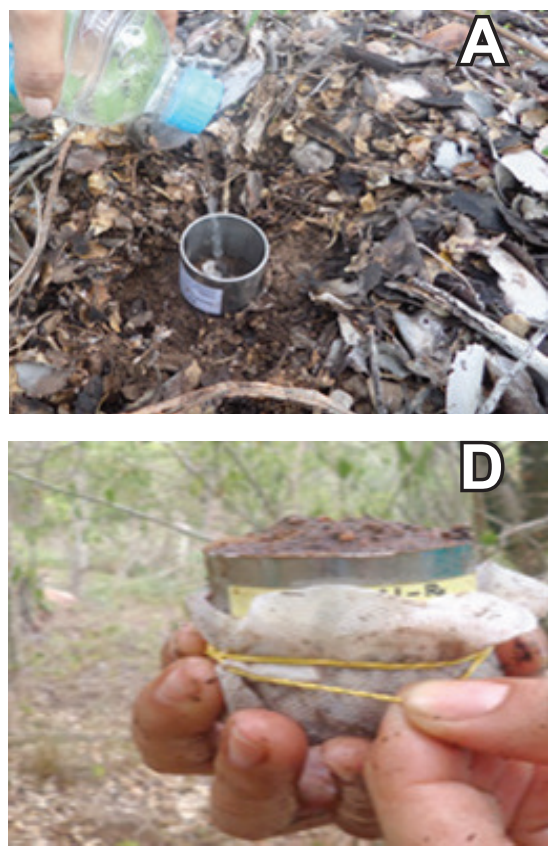
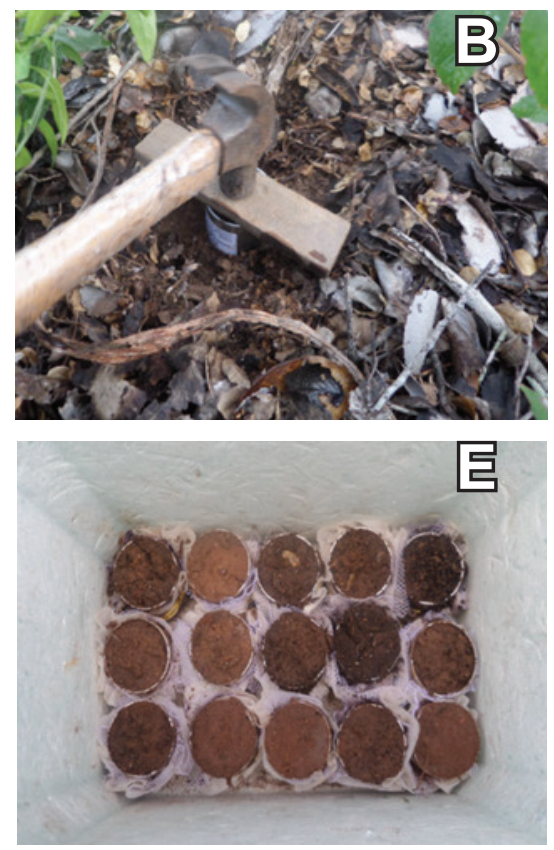
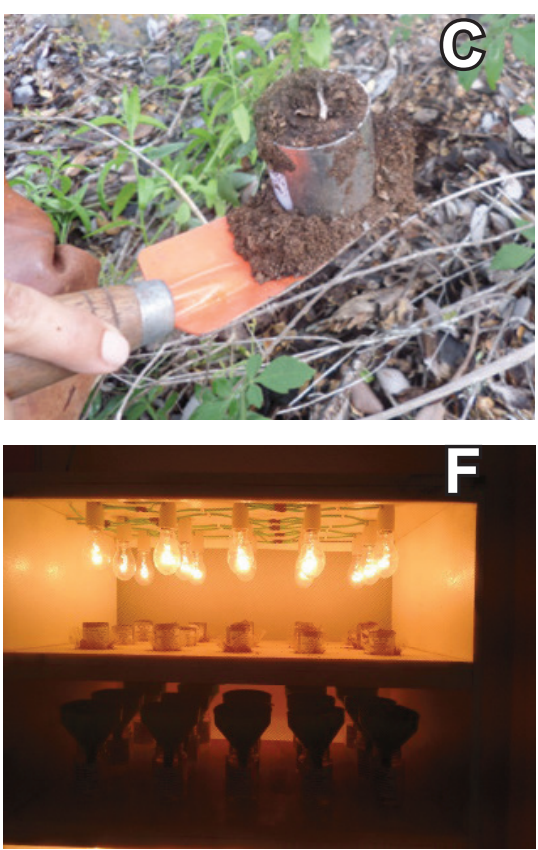
Os organismos foram armazenados em recipientes de vidro com solução de álcool $70 \%$. A mesofauna capturada com comprimento entre 0,2 e 2,0 $\mathrm{mm}$ foi quantificada e identificada com auxílio de microscópio estereoscópio e chave de identificação (Swift et al., 1979).

A mesofauna edáfica foi avaliada quantitativamente pela abundância (número de indivíduos) e qualitativamente pela diversidade, utilizando o Índice de Shannon, pela equação: $H=-\sum$ pi. logpi, em que: pi=ni/N; ni=densidade de cada grupo; $\mathrm{N}=\Sigma$ ni. $\mathrm{O}$ Índice de Pielou foi definido pela equação: $e=H / \log S$, em que: H=Índice de Shannon; S=Número de espécies ou grupos. A constância dos grupos taxonômicos foi determinada de acordo com Silveira Neto et al. (1976), pela equação $c=(p \times 100) / P$, em que: $\mathrm{p}=$ número de coletas contendo o grupo; $\mathrm{P}=$ número total de coletas realizadas. De acordo com os percentuais obtidos, os grupos foram classificados nas categorias: espécies constantes (presentes em mais de $50 \%$ das coletas), espécies acessórias (presentes em 25 a $50 \%$ das coletas) e espécies acidentais (presentes em menos de $25 \%$ das coletas) (Bodenheimer, 1995).

\section{RESULTADOS E DISCUSSÃO}

No período estudado foram contabilizados 84 organismos da mesofauna edáfica, distribuídos em nove grupos taxonômicos (Figura 2). A riqueza foi elevada, pois comparando com o trabalho de Almeida et al. (2013), em doze coletas detectaram 13 grupos taxonômicos em pesquisa realizada no Semiárido Paraibano.

Figura 2. Percentagem (\%) dos grupos taxonômicos da mesofauna edáfica em Santana do Ipanema, Alagoas

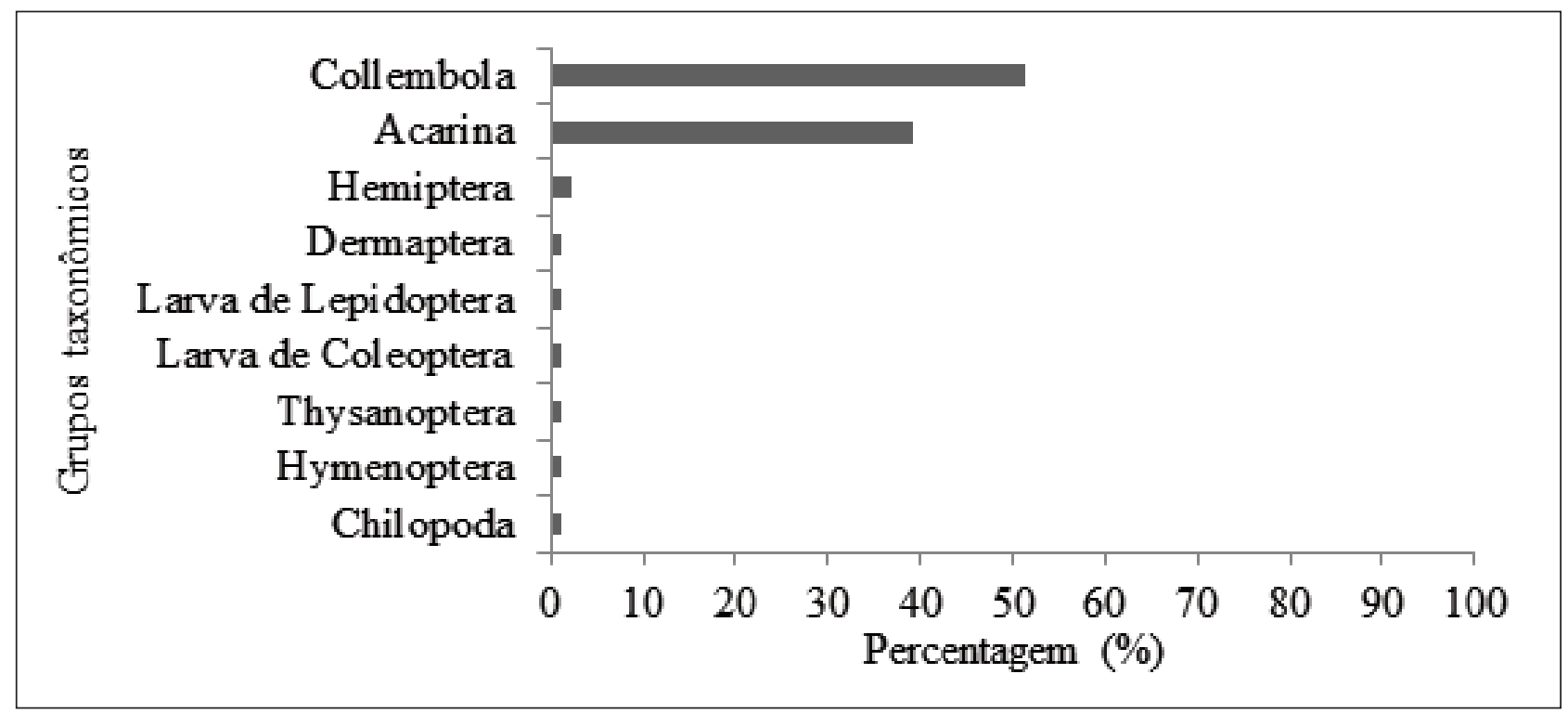

Observou-se o elevado número de indivíduos dos grupos Collembola $(51,19 \%)$ e Acarina $(39,29 \%)$ (Figura 2) em relação aos demais grupos.

A abundância do grupo Collembola está associada a sua capacidade de se multiplicar e crescer rapidamente (Antoniolli et al., 2013). Esse grupo se desenvolve melhor em ambientes de vegetação nativa, onde as condições como variedade de espécies vegetais e compostos orgânicos no solo permitem uma maior diversidade dos organismos edáficos (Rief et al., 2010), como é o caso da área estudada.

De acordo com Formiga (2014) o aumento ou decréscimo no número de indivíduos está atribuído às características oportunistas de determinados organismos ao ambiente de Caatinga, podendo apresentar comportamento sazonal.

Em relação à constância, verificou-se que os grupos Acarina e Collembola foram classificados como constantes por estarem presentes nas quatro coletas realizadas. Os demais grupos taxonômicos ficaram na categoria acessória, presentes em 25 a 50\% das coletas (Tabela 1). Tem sido hipotetizado que espécies constantes podem ser residentes, as acessórias podem também ser residentes, mas apresentam flutuações e as acidentais são aquelas imigrantes, as quais entrariam esporadicamente para se alimentarem ou se reproduzirem (Santos, 1999). 
Tabela 1. Percentual da constância (C\%) e categoria da constância dos grupos taxonômicos da mesofauna edáfica

\begin{tabular}{ccc}
\hline Grupos taxonômicos & C (\%) & Categoria \\
\hline Acarina & 100 & Constante \\
Chilopoda & 25 & Acessória \\
Collembola & 100 & Constante \\
Dermaptera & 25 & Acessória \\
Hemiptera & 25 & Acessória \\
Hymenoptera & 25 & Acessória \\
Larva de Coleoptera & 25 & Acessória \\
Larva de Lepidoptera & 25 & Acessória \\
Thysanoptera & 25 & Acessória \\
\hline
\end{tabular}

Avaliando-se a diversidade e uniformidade pelos índices de Shannon $(\mathrm{H})$ e Pielou $(e)$ registradas durante as quatro coletas, constatou-se que os grupos Collembola $(H=0,29 ; \quad e=0,18)$ e Acarina $(H=0,41 ; e=0,27)$ apresentaram os menores valores (Figuras 3A e 3B). Em estudo realizado no Semiárido
Paraibano foi constatado os menores valores para os grupos Acarina e Collembola (Araujo et al., 2013). $O$ índice de Shannon $(\mathrm{H})$ varia de 0 a 5 , sugerindo que o declínio de seus valores é o resultado de uma maior dominância de grupos em detrimento de outros (Begon et al., 1996).

Figura 3. Índices de Shannon-H (A) e Pielou-e (B) para os grupos taxonômicos da mesofauna edáfica

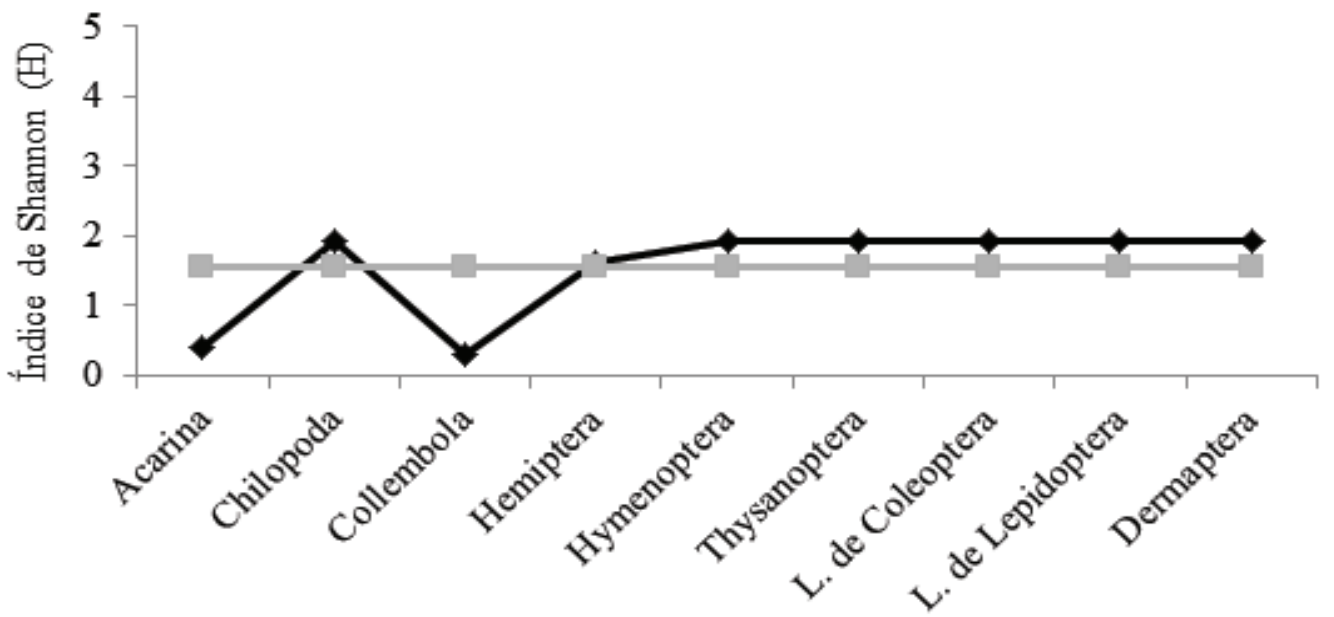

Grupos Taxonômicos

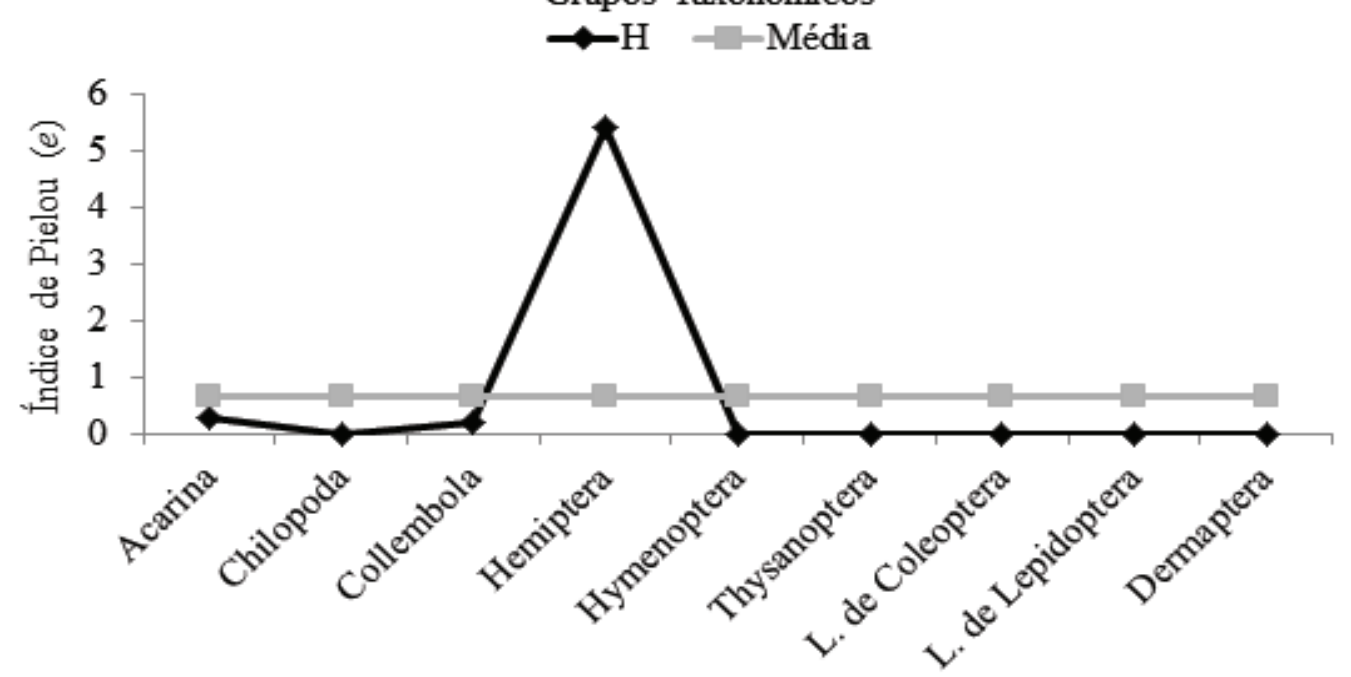


Diante dos resultados obtidos na pesquisa, pode-se concluir que os grupos taxonômicos mais dominantes na área de Caatinga, confirmado pelos baixos Índices de Shannon e Pielou são Collembola e Acarina, classificados como constantes em relação aos demais grupos que são inseridos na categoria acessória e que o remanescente de Caatinga estudado encontrase preservado fornecendo condições favoráveis à dinâmica dos organismos da mesofauna edáfica.

\section{AGRADECIMENTOS}

À Universidade Federal de Alagoas pela concessão da bolsa de estudo. À EMATER pela concessão do uso da área experimental.

\section{REFERÊNCIAS BIBLIOGRÁFICAS}

ALAGOAS. Perfil Municipal: Santana do Ipanema. 3. ed. SEPLANDE/AL, Maceió, 2015. 24.

ALMEIDA, M.A.X.; SOUTO, J.S.; SOUTO, P.C. Composição e sazonalidade da mesofauna edáfica do solo do semiárido paraibano. Revista Verde de Agroecologia e Desenvolvimento Sustentável, 2013, 8, 4, 214-222.

ANTONIOLLI, Z.I.; REDIN, M., SOUZA, E.L. de; POCOJESKI, E. Metais pesados, agrotóxicos e combustíveis: efeito na população de colêmbolos no solo. Revista Ciência Rural, 2013, 43, 6, 992-998.

ARAUJO, K.D.; DANTAS, R.T.; ANDRADE, A.P. de; PARENTE, H.N.; PAZERA JÚNIOR, E. Dinâmica da mesofauna edáfica em função das estações seca e chuvosa em áreas de caatinga sob pastejo. Brazilian Geographical Jounal: Geosciences and Humanities Research Medium, 2013, 4, 2, 663-679.

BEGON, M.; HARPER, J.L.; TOWNSEND, C.R. Ecology: individuals, populations and communities. 3. ed. Oxford: Blackwell Science, 1996. 1068.

BERUDE, M.C.; GALOTE, J.K.B.; PINTO, P.H.; AMARAL, A.A. do. A mesofauna do solo e sua importância como bioindicadora. Enciclopédia Biosfera, 2015, 11, 22, 14-28.
BODENHEIMER, F.S. Precis d'ecologie animale. 1 ed. Paris: Payout, 1995, 315.

EMBRAPA - Empresa Brasileira de Pesquisa Agropecuária. Zoneamento agroecológico de Alagoas: levantamento de reconhecimento de baixa e média intensidade dos solos do Estado de Alagoas. 1. ed. Recife: EMBRAPA, 2012. 238.

FORMIGA, L.D.A.S. Organismos edáficos, cinética do $\mathrm{CO}_{2}$ e herbivoria em áreas de Caatinga sob pastejo caprino. Tese de Doutorado, Universidade Federal da Paraíba, 2014. 122.

KORASAKI, V.; MORAIS, J.W. DE; BRAGA, R.F. In: Moreira, F.M.S.; CARES, J.E.; ZANETTI, R.; STÜMER, S.L. (Eds.). O ecossistema solo: componentes, relações ecológicas e efeitos na produção vegetal. 1 ed. Lavras:

Editora da UFLA, 2013. 185-200.

RIEFF, G.G.; MACHADO, R.G.; STROSCHEIN, M.R.D.; SACCOL DE SÁ, E.L. Diversidade de famílias de ácaros e colêmbolos edáficos e cultivo de eucalipto e áreas nativas. Revista Brasileira de Agrociência, 2010, 16, 1, 57-61.

SANTOS, G.B. Estrutura de comunidades de peixes de reservatórios do sudeste do Brasil, localizados nos rios Grande e Paranaíba, bacia do Alto Paraná. Tese de Doutorado, Universidade Federal de São Carlos, 1999. 166.

SILVEIRA NETO, S.; NAKANO, O.; BARBIN, D.; VILLA NOVA, N. A. Manual de ecologia dos insetos. 1 ed. São Paulo: Ceres, 1976, 429.

SWIFT, M.J.; HEAL, O.W.; ANDERSON, J.M. Decomposition in terrestrial ecosystems: studies in ecology. 5. ed. Oxford: Blackwell Scientific, 1979. 238. 